\title{
Thermodynamic analysis of pumped thermal electricity storage
}

\author{
Alexander White ${ }^{a^{*}}$, Geoff Parks ${ }^{a}$ and Christos N. Markides ${ }^{b}$ \\ ${ }^{a}$ Cambridge University Engineering Department, Trumpington Street, Cambridge CB2 1PZ, United Kingdom \\ ${ }^{\mathrm{b}}$ Department of Chemical Engineering and Chemical Processing, Imperial College, London SW7 2AZ, United Kingdom \\ * Corresponding author. Email: ajw36@cam.ac.uk; Tel. +44(0) 1223 765310; Fax.+44(0) 1223765311
}

\begin{abstract}
The increasing use of renewable energy technologies for electricity generation, many of which have an unpredictably intermittent nature, will inevitably lead to a greater need for electricity storage. Although there are many existing and emerging storage technologies, most have limitations in terms of geographical constraints, high capital cost or low cycle life, and few are of sufficient scale (in terms of both power and storage capacity) for integration at the transmission and distribution levels. This paper is concerned with a relatively new concept which will be referred to here as Pumped Thermal Electricity Storage (PTES), and which may be able to make a significant contribution towards future storage needs. During charge, PTES makes use of a high temperature-ratio heat pump to convert electrical energy into thermal energy which is stored as 'sensible heat' in two thermal reservoirs, one hot and one cold. When required, the thermal energy is then converted back to electricity by effectively running the heat pump backwards as a heat engine. The paper focuses on thermodynamic aspects of PTES, including energy and power density, and the various sources of irreversibility and their impact on round-trip efficiency.
\end{abstract}

Keywords electricity storage; thermal energy storage; irreversibility; heat transfer

\section{Introduction}

In order to meet the UK's target for the EU Renewable Energy Directive, forecasts suggest that more than $30 \%$ of electricity may need to come from renewable sources by 2020 [1]. Much of this is likely to be in the form of wind generation, which produces supplies that are highly variable, uncontrollable and to some extent unpredictable. Combatting such variability will be one of the major challenges for the high penetration of renewable technologies and is likely to require a combination of demand side management, reserve capacity and, especially, increased energy storage. The scale of the problem is discussed by MacKay [2] who suggests that up to $1200 \mathrm{GWh}$ of storage may be required in the UK if a quarter of today's electricity demand is to be met by wind energy. To put this into perspective, the UK's current inventory of storage facilities (nearly all of which is pumped hydro) can store about 30 $\mathrm{GWh}$, and its scope for extension is severely limited by geographic and planning constraints. There is thus a pressing need to investigate new, smaller-footprint alternatives.

\subsection{Other storage technologies}

The list of possible, alternative storage methods is extensive and includes: flywheels, super capacitors, batteries and flow batteries, Compressed Air Energy Storage (CAES), Superconducting Magnetic Energy Storage (SMES) and Thermal Energy Storage (TES) in its various forms. A review of many of these technologies is given by Chen et al. [3]. Some (e.g., flywheels and super capacitors) have very high efficiency, fast response and high power density, but are only able to supply power for short durations. They are therefore most appropriate for power quality management applications - e.g., bridging short-duration interruptions and providing voltage and frequency support during rapid supply or demand swings. For energy management applications - e.g., levelling daily demand fluctuations and smoothing the output from intermittent renewable sources - CAES is probably the leading competitor to Pumped Hydro Storage (PHS), but it too suffers geographic limitations since large, robust caverns are required for the storage of air at pressures up to 100 bar. Amongst the other candidate technologies, few are currently able to provide both multi-megawatt scale capacity and long-duration (i.e., hours) discharge; many are expensive (often several times the cost of open-cycle standing reserve gas turbines in terms of $£ / \mathrm{kW}$ installed capacity), and many make use of hazardous, toxic or scarce materials. 


\subsection{Description of the PTES system}

The subject of the current paper, PTES, is a recent concept which is currently being developed in the UK (see for example ref. [4]) and is also being considered in France [5]. We include a brief description of a PTES system here since it is a not widely known technology. The general principle of operation is straightforward and is illustrated in Fig. 1. The main components are a compressor (C), an expander (E) and two thermal stores: one hot (HS) and one cold (CS). The latter are insulated cylindrical vessels containing an appropriate thermal storage medium: e.g., a packed bed of pebbles or gravel, or a uniform matrix of ceramic. Energy is stored as 'sensible heat', enabling efficient integration with the thermodynamic cycle and avoiding the 'pinch-point' difficulties associated with phase-change storage methods. $\mathrm{C}$ and $\mathrm{E}$ may be either turbomachinery or reciprocating devices. They are mechanically coupled and linked to a motor-generator. The heat exchangers (HX1 and HX2) serve mainly to reject heat from the cycle (via cooling water), this being required to combat the effects of system irreversibilities.
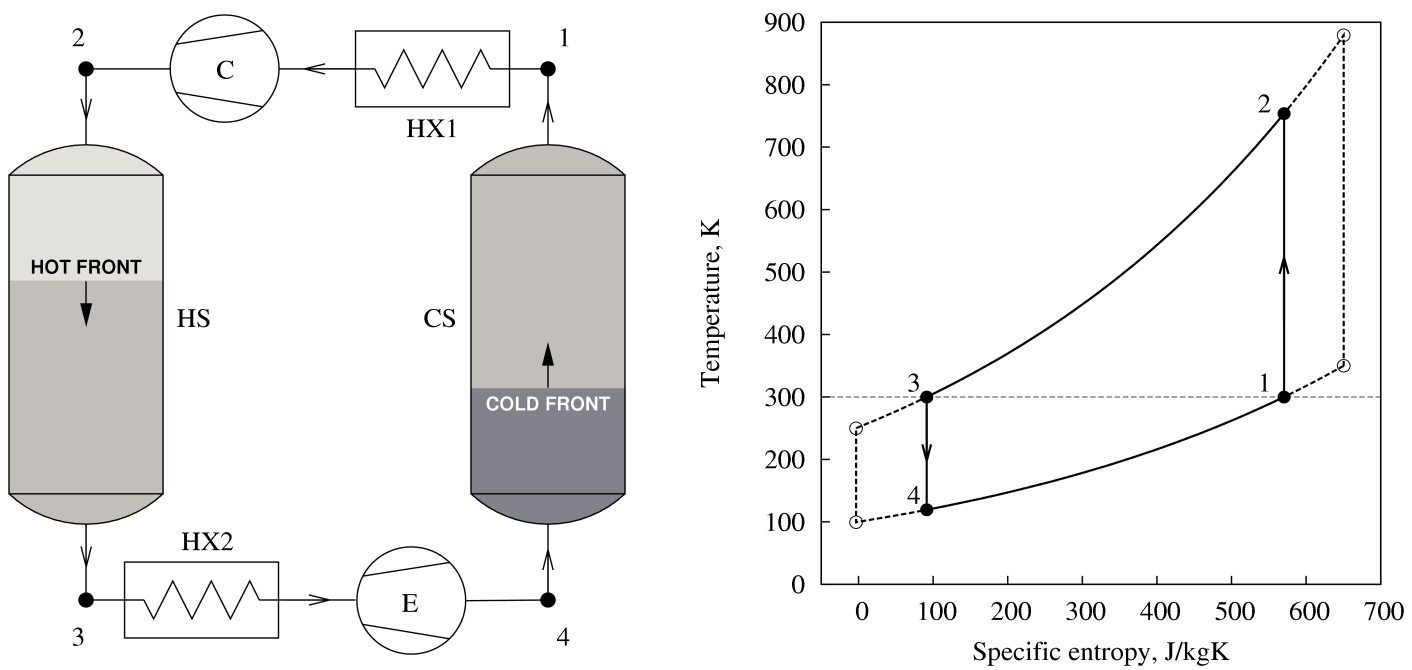

Figure 1: Layout of a PTES system and $T-s$ diagram of the ideal cycle shown during charge

The working fluid is a gas (Argon) which flows in the direction indicated by the arrows during charge, but is reversed for discharge. During charge, the system operates as a high temperature-ratio heat pump, using electrical energy to extract heat from CS and deliver heat to HS. This takes place by the progression of hot and cold fronts in the stores, as indicated in the layout figure. During discharge, the processes are reversed so that the device operates as a heat engine: heat is returned from the hot to the cold store and electrical energy is retrieved. (Note that if reciprocating devices are used, the flow directions of $\mathrm{C}$ and $\mathrm{E}$ can be reversed during discharge such that they become an expander and compressor respectively.) The heat engine and heat pump operate on the Joule-Brayton and reverse Joule-Brayton cycles respectively, the temperature-entropy $(T-s)$ diagram for which is shown on the right of the figure for the case of ideal (reversible) processes.

Although PTES is in the early stages of development, it is based on well-established technologies and it is therefore possible to estimate its likely performance (round-trip efficiency, storage density and capital cost) with a reasonable level of confidence. Initial estimates suggest that it may well be able to compete with CAES and PHS, but without the associated geographic limitations. The objectives of the present paper are to provide the thermodynamic background to estimates of PTES system performance, focusing in particular upon how the various sources of loss impinge upon round-trip efficiency and thereby pointing towards strategies for optimisation.

\section{Simplified cycle calculations}

The potential success of PTES hinges upon obtaining a satisfactory round-trip efficiency (i.e., electrical energy output/electrical energy input) whilst simultaneously keeping capital costs as low as possible. Ultimately, successful design will require comprehensive system modelling, taking into account a wide variety of economic, thermodynamic, mechanical, electrical and other factors. Such modelling is underway, but in anticipation of its completion we present here instead a simplified 
model which, nonetheless, provides a basic understanding of how the main operating conditions and loss parameters influence performance. The focus is upon thermodynamic aspects of PTES since electrical and mechanical issues are common to several other storage methods.

\subsection{Energy and power density}

For a given technology, the capital cost per unit energy storage capacity (in $£ / k W h$ ) and per unit power capacity (in $£ / \mathrm{kW}$ ) will depend inversely on the energy storage density, $\rho_{\mathrm{E}}$, and power density, $\rho_{\mathrm{P}}$, respectively. These are thus key performance parameters for any storage method. We start by considering a fully reversible PTES system, as shown in the $T-S$ diagram of Fig. 1 . The processes involved are the same as for the standard Joule-Brayton cycle (isentropic compression and expansion and isobaric heat addition and rejection), but traversed in the opposite direction during charge. The round-trip efficiency for the reversible cycle is unity by definition, irrespective of the cycle pressures and temperatures, but it is nonetheless useful to consider this case as it provides reasonable estimates for $\rho_{\mathrm{E}}$ and $\rho_{\mathrm{P}}$.

Defining $\rho_{\mathrm{E}}$ as the average energy stored in the reservoirs per unit volume of storage medium, and $\rho_{\mathrm{P}}$ as the power output per unit volumetric flow rate of the working fluid, straightforward analysis gives:

$$
\begin{aligned}
& \rho_{\mathrm{E}}=\frac{1}{2} \rho_{\mathrm{s}} c_{\mathrm{s}}\left\{\left(T_{2}-T_{3}\right)-\left(T_{1}-T_{4}\right)\right\}=\frac{1}{2} \rho_{\mathrm{s}} c_{\mathrm{s}} T_{1}(\tau-1)(1-\theta / \tau) \\
& \rho_{\mathrm{P}}=\rho_{\mathrm{g} 1} c_{\mathrm{pg}}\left\{\left(T_{2}-T_{1}\right)-\left(T_{3}-T_{4}\right)\right\}=(\tau-1)(1-\theta / \tau) \times \gamma p_{1} /(\gamma-1)
\end{aligned}
$$

where $\rho_{\mathrm{s}}$ and $\rho_{\mathrm{g} 1}$ are the storage medium and gas density respectively (at state 1 for the latter), $c_{\mathrm{s}}$ and $c_{\mathrm{pg}}$ are the corresponding specific heat capacities (isobaric for the gas), $\tau=T_{2} / T_{1}=T_{3} / T_{4}$ is the compressor and expander temperature ratio, and $\theta=T_{3} / T_{1}$ is the ratio between the hot and cold reservoir temperatures when discharged. The following may be deduced from these expressions:

i. Both the energy and power density are monotonically increasing functions of the temperature ratio $\tau$, which depends on the pressure ratio and the isentropic index $(\gamma)$ of the gas.

ii. For a given pressure ratio (or $\tau$ ), $\rho_{\mathrm{E}}$ and $\rho_{\mathrm{P}}$ are both increased by reducing $T_{3}$ or increasing $T_{1}$. This is also shown by the dashed line in the $T-s$ diagram of Fig. 1 which encloses a larger area.

iii. The power density may also be increased by raising the overall system pressure. The factor $\gamma /(\gamma-1)$ also has a significant influence and takes the value $5 / 2$ for monatomic and $7 / 2$ for diatomic gases.

Table 1:

Energy and power densities for a few storage technologies

\begin{tabular}{|l|c|c|c|c|}
\hline & PTES $^{\mathbf{a}}$ & PHS $^{\mathbf{b}}$ & CAES $^{\mathbf{c}}$ & GT $^{\text {d }}$ \\
\hline Storage medium: & gravel & water & air & - \\
\hline Working fluid: & Argon & water & air & air \\
\hline Energy density, $\rho_{\mathrm{E}}\left(\mathrm{kWh} / \mathrm{m}^{3}\right)$ & 50 & 1.4 & 10 & - \\
\hline Power density, $\rho_{\mathrm{P}}\left(\mathrm{kW} / \mathrm{m}^{3} \mathrm{~s}^{-1}\right)$ & 240 & 5000 & - & 330 \\
\hline
\end{tabular}

${ }^{\mathrm{a}}$ with $T_{1}=T_{3}=300 \mathrm{~K}, p_{1}=1 \mathrm{bar}, \tau=2.58$ (solid line in Fig. 1 and the approximate conditions for the system in ref. [4]).

${ }^{\mathrm{b}}$ with altitude drop $\Delta h=500 \mathrm{~m}$; both $\rho_{\mathrm{P}}$ and $\rho_{\mathrm{E}}$ are given by $\rho_{\mathrm{H} 2 \mathrm{O}} g \Delta h$

${ }^{c}$ with storage at 100 bar; see ref. [6] for the energy density expression for CAES.

${ }^{\mathrm{d}}$ with $\tau=2.05$ (pressure ratio $\sim 12$ ) and $T_{\max }=900^{\circ} \mathrm{C}$.

Table 1 shows estimates of the energy and power densities for PTES compared with pumped hydro and compressed air storage. In all cases the estimates are based on ideal or reversible systems. Note that CAES is not a pure storage technology because it operates as part of a gas turbine cycle so the power density of a low-spec gas turbine is given to provide an approximate comparison. Comparisons between technologies should be treated with caution, but it is nonetheless reasonable to conclude that PTES has very good energy density and a power density that is not too much below that of a low-spec gas turbine.

\subsection{Approximate susceptibility to irreversibility}

System irreversibilities tend to reduce expansion work outputs and increase compressor work inputs during both charge and discharge. An approximate estimate of how operating conditions influence the 
round-trip efficiency for the real (irreversible) system can thus be obtained by scaling compression work by $1 / \eta$ and expansion work by $\eta$, where $\eta$ may be interpreted here as an average isentropic efficiency. The round-trip efficiency is then given by:

$\chi=\frac{\eta\left(T_{2}-T_{1}\right)-\left(T_{3}-T_{4}\right) / \eta}{\left(T_{2}-T_{1}\right) / \eta-\eta\left(T_{3}-T_{4}\right)}=\frac{R \eta^{2}-1}{R-\eta^{2}}$

where $R=T_{1} / T_{4}=T_{2} / T_{3}=\tau / \theta$, and $T_{1}$ to $T_{4}$ represent the temperatures for the reversible rather than the real cycle. Eq. (3) shows that, for a given value of $\eta$, the round-trip efficiency is a continuously increasing function of the ratio $R$. This remains approximately true even for a more realistic treatment of system losses (see ref. [6]) and is merely a reflection of the fact that $R$ is the ratio between the compression and expansion work (during charge); losses therefore have a smaller impact on the net work as $R$ is increased. The ratio $R$ may be increased by increasing the pressure ratio, decreasing the hot reservoir discharged temperature, $T_{3}$, or increasing the cold reservoir discharged temperature, $T_{1}$, all of which are consistent with improving the energy and power densities. High pressure ratios, however, lead to high storage costs since HS needs to be pressurised. It is for this reason that Argon is proposed as the working fluid, rather than air, since the same value of $\tau$ can be achieved at a lower pressure ratio due to Argon's higher isentropic index.

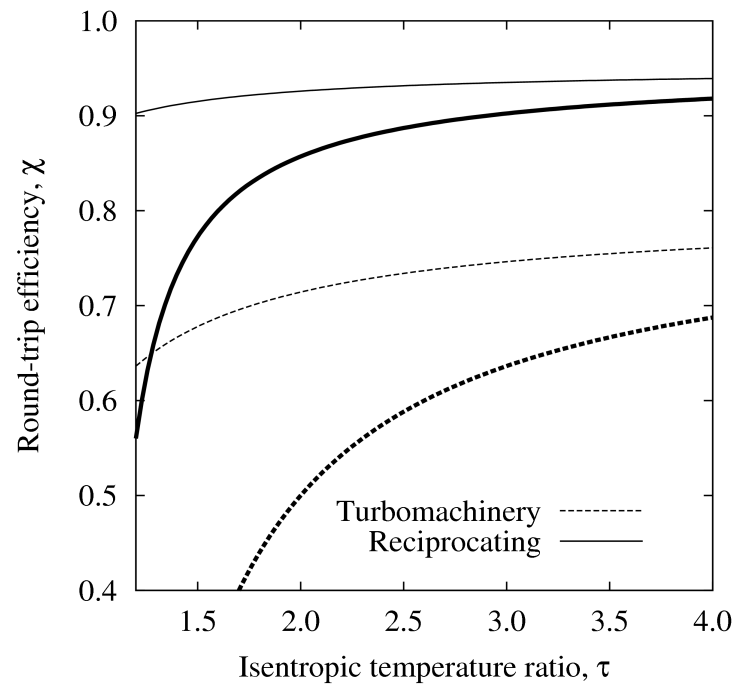

(a) approximate round-trip efficiency

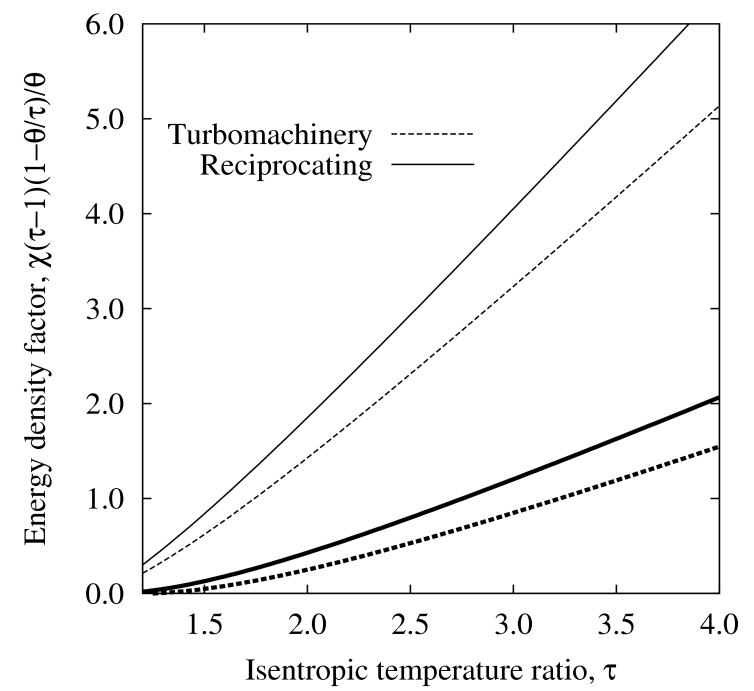

(b) storage energy density factor

Figure 2: Approximate round-trip efficiency and energy density for $\theta=1$ (bold) and $\theta=0.4$ (faint)

Figure 2 shows the approximate round-trip efficiency based on Eq. (3) and the temperature dependent factor of $\rho_{\mathrm{E}}$ (i.e., $(\tau-1)(1-\theta / \tau) / \theta$, which is equivalent to Eq. (1) if $T_{3}$ is fixed, as discussed further in section 2.3.3). Each quantity is plotted versus $\tau$ and the energy density factor has been multiplied by $\chi$ to give a better representation of the recoverable energy. Two sets of curves are shown, one for a turbomachinery-based system, for which $\eta^{2}=0.80$, and one for a system based on reciprocating devices, for which the assumed value of $\eta^{2}$ is 0.95 . In each case, curves are plotted for $\theta=1$ (bold) and $\theta=0.4$ (faint). (These two values of $\theta$ bracket the likely practical range and intermediate values provide curves that are intermediately located - i.e., the dependence on $\theta$ is monotonic.) The graphs clearly show the benefits of reducing $\theta$, especially for the turbo-machinery-based system. Such benefits would not be realised, however, if the maximum and minimum temperatures within the cycle were to be constrained, as discussed below.

Despite being based on the same thermodynamic cycle, it is clear from the figures that trends are quite different to those of a gas turbine. For example, gas turbines have optimal pressure ratios for both efficiency and power density, partly as a consequence of the turbine inlet temperature being limited by material constraints. In practice, there are likely to be limits on $T_{2}$ and $T_{4}$ in a PTES system for similar reasons, and if these two temperatures are fixed, then it may be shown from Eq. (1) that the 
maximum energy density occurs for $\tau^{2}=\left(T_{2} / T_{4}\right)$ and $\theta=1$, provided losses are small. However, optimisation cannot be properly undertaken without considering a range of extra-thermodynamic factors, particularly those associated with capital cost and materials issues.

\subsection{Detailed loss analysis}

The aim of this section is to describe the various sources of loss and provide preliminary estimates of their impact on round-trip efficiency. Several of the loss parameters (for example, the compression and expansion efficiencies for reciprocating devices) are difficult to specify with great certainty at this stage. The approach adopted here is therefore to determine the sensitivity of the round-trip efficiency to these loss parameters. The chief sources of loss are as follows:

i. Electrical and mechanical losses. These lie outside the scope of the present paper which focuses instead upon thermodynamic factors. However, it is worth noting that electrical/mechanical energy conversion can be achieved very efficiently; $\eta_{\mathrm{e}} \geq 97 \%$ is not unreasonable for large devices. Mechanical losses are more uncertain and are likely to be greater for reciprocating devices than for turbomachinery. In total, electrical and mechanical losses might reduce the round-trip efficiency by $10 \%$ or more.

ii. Compression and expansion losses. For turbomachinery these stem chiefly from viscous effects and are quantified here by specifying a polytropic (infinitesimal stage) efficiency. In the case of reciprocating devices, compression and expansion occur relatively slowly such that there may be significant heat transfer with the cylinder walls. It is important to distinguish between net heat exchange with the surroundings and the periodic exchange to and from the walls which, although globally adiabatic, is an irreversible process and incurs an entropy rise. Other losses result from pressure drops through valves and from mixing of fresh charge with residual gas. Reciprocating devices are thus modelled here by specifying (a) a polytropic efficiency to account for thermal dissipation and mixing, and (b) a heat loss (or gain) factor, $\alpha$, defined as the ratio between net heat transfer and work transfer. Valve losses are included in the pressure loss factor described below.

iii. Pressure losses. These occur through piping, valves (as described above), diffusers (especially for turbomachinery) and through the hot and cold reservoirs. In terms of impact on the round-trip efficiency, it is the fractional pressure loss, $f=\Delta p / p$, in each device that is most relevant since this is proportional to the entropy increase and hence to the lost work. Individual values of $f$ are expected to be small and so can be summed to give an overall pressure loss factor $F$.

iv. Thermal reservoir losses. Aside from pressure losses, there are two other loss mechanisms within the thermal stores. Firstly, heat exchange with the exterior means that the available energy stored within each reservoir is depleted with time. This is quantified by specifying an energy loss factor, $\varepsilon$, defined below. Secondly, the process of heat transfer between the gas and storage medium requires a finite temperature difference and thus incurs a net entropy increase. The associated losses are quite complex and depend on the mode of operation of the storage system (e.g., regular periodic cycling or long-term storage). Further discussion is given in section 2.3.4 below.

Inclusion of the various losses modifies the $T-s$ diagrams as shown in Fig. 3 (the condition points are as indicated in the layout of Fig. 1). Entropy increases during compression and expansion and various pressure losses mean that the charge and discharge cycles are no longer coincident and, as expected, the work output falls below the work input during charge. Heat must therfore be rejected from the cycle. If the discharge pressure ratio is the same as that for charging (Fig. 3a) then the compressor delivery temperature, $T_{3}{ }^{\prime}$, lies above $T_{3}$ and so heat rejected via HX2 (see Fig. 1) such that HS can be restored to its initial, discharged state. Likewise, heat must also be rejected between states $1^{\prime}$ and 1 via HX1 in order to return CS to its initial state. Alternatively, as shown in Fig. 3(b), a lower discharge pressure ratio can be used such that $T_{3}{ }^{\prime}=T_{3}$. In this case all the heat is rejected between states $1^{\prime}$ and 1 in HX1. In fact, the optimal pressure ratio lies somewhere between these two cases, but the variation in efficiency is usually small unless compression and expansion losses are very large. Note also that using a lower discharge pressure ratio and then bypassing HX1 enables $\theta=T_{3} / T_{1}$ to be reduced between successive cycles in order to obtain the benefits described in sections 2.1 and 2.2. 


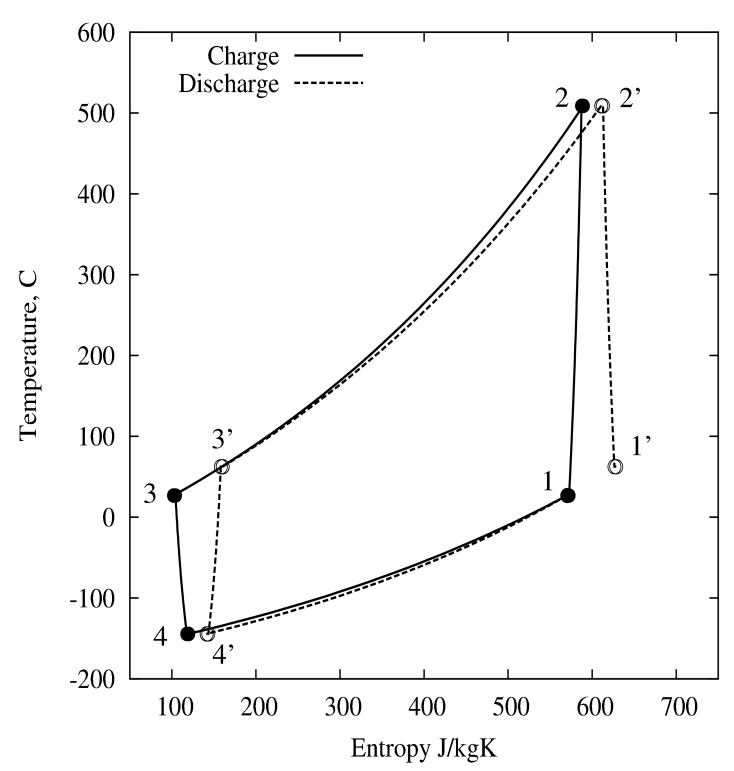

(a) with same discharge and charge pressure ratio

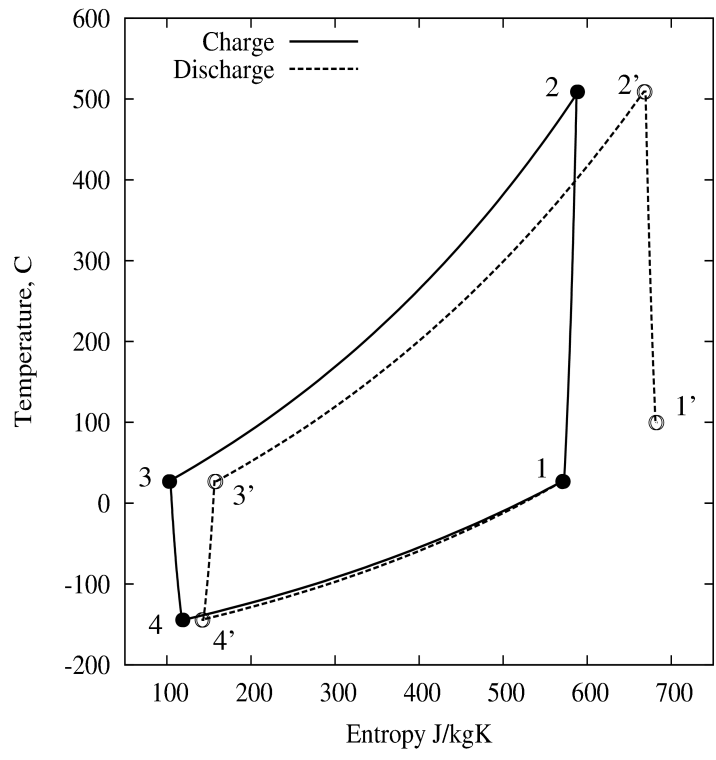

(b) with lower discharge pressure ratio

Figure 3: Temperature-entropy diagrams for irreversible PTES cycles

\subsubsection{General expression for round-trip efficiency}

Ignoring reservoir losses for the time being, inspection of Fig. 3 shows that the thermodynamic roundtrip efficiency can be expressed as:

$$
\chi=\frac{w_{\mathrm{e}}^{\prime}-w_{\mathrm{c}}^{\prime}}{w_{\mathrm{c}}-w_{\mathrm{e}}}=\frac{\left(T_{2}^{\prime}-T_{1}^{\prime}\right) /\left(1-\alpha_{\mathrm{e}}^{\prime}\right)-\left(T_{3}^{\prime}-T_{4}^{\prime}\right) /\left(1-\alpha_{\mathrm{c}}^{\prime}\right)}{\left(T_{2}-T_{1}\right) /\left(1-\alpha_{\mathrm{c}}\right)-\left(T_{3}-T_{4}\right) /\left(1-\alpha_{\mathrm{e}}\right)}
$$

where the primes denote quantities during discharge. The $1 /(1-\alpha)$ factors reflect the occurrence of both heat and work transfer during each of the compression and expansion processes (see ref. [7]).

Eq. (4) can be simplified by first noting that (in the absence of heat losses from the reservoirs) $T_{2}{ }^{\prime}=T_{2}$ and $T_{4}{ }^{\prime}=T_{4}$. We will also assume that $T_{3}{ }^{\prime}=T_{3}$, corresponding to Fig. 3(b) above. Finally, we will assume that $\alpha_{c}=\alpha_{e}=\alpha$ during charge, and $\alpha_{c}{ }^{\prime}=\alpha_{e}{ }^{\prime}=-\alpha$ during discharge. This reflects the fact that heat transfer will be to the surroundings for hot components and from the surroundings for cold ones. With these simplifications, Eq. (4) becomes:

$\chi=\left(\frac{1-\alpha}{1+\alpha}\right)\left\{1-\frac{\tau_{\mathrm{c}} / \tau_{\mathrm{e}}^{\prime}-1}{\left(\tau_{\mathrm{c}}-1\right)-\theta\left(1-1 / \tau_{\mathrm{e}}\right)}\right\}$

The various temperature ratios, $\tau$, are related to the corresponding pressure ratios, $\beta$, by expressions of the form $\tau=\beta^{n}$, where $n=(\gamma-1)\left(1-\alpha_{\mathrm{e}}\right) \eta_{\mathrm{e}} / \gamma$ for expanders and $n=(\gamma-1)\left(1-\alpha_{\mathrm{c}}\right) / \eta_{\mathrm{c}} \gamma$ for compressors (see ref. [7] for derivation). Note that a $10 \%$ heat loss from a compressor combined with a $90 \%$ polytropic efficiency gives rise to an isentropic process, but the effect is quite different to that of adiabatic reversible compression. Pressure losses result in expanders seeing a lower pressure ratio than compressors such that, for example, $\beta_{\mathrm{e}}=(1-F) \beta_{\mathrm{c}}$.

\subsubsection{Impact of pressure, compression and expansion losses on efficiency}

A useful indication of how the various losses impact upon round-trip efficiency is given by the partial derivatives of $\chi$ with respect to each of the loss parameters in turn. The algebra is considerably simplified by evaluating these derivatives at loss-free conditions (i.e., $\eta=1, F=\alpha=0$ ). Straightforward but lengthy manipulations then give:

$$
S_{\alpha}=\frac{\partial \chi}{\partial \alpha}=-2
$$




$$
\begin{aligned}
& S_{F}=\frac{\partial \chi}{\partial F}=\frac{-2(\gamma-1) / \gamma}{(\tau-1)(1-\theta / \tau)} \\
& S_{\eta}=\frac{\partial \chi}{\partial \eta}=\frac{4 \ln \tau}{(\tau-1)(1-\theta / \tau)}
\end{aligned}
$$

where $\tau$ is the temperature ratio under loss-free conditions, and for Eq. (8) it has been assumed that all compressions and expansions have the same polytropic efficiency, $\eta$. Comparison with numerical cycle calculations (e.g., [6]) suggest a reasonable estimate of overall cycle loss can be obtained from a linear combination of the loss contributions based on these sensitivity parameters, provided $\alpha, F$ and $(1-\eta)$ are each only a few percent.

\subsubsection{Impact of reservoir heat leakage on efficiency}

The effect of heat leakage to or from the reservoirs (as opposed to losses due to irreversible heat transfer) can be assessed by considering the total availability stored in the HS and CS. If each reservoir has a thermal capacity of $M_{\mathrm{s}} c_{\mathrm{s}}$, then this is given by:

$$
B / M_{\mathrm{s}} c_{\mathrm{s}}=\left(T_{2}-T_{3}\right)-T_{0} \ln \left(T_{2} / T_{3}\right)+\left(T_{4}-T_{1}\right)-T_{0} \ln \left(T_{4} / T_{1}\right)
$$

where $T_{0}$ is the temperature of the environment. (Note that for a reversible PTES system, $T_{4} / T_{1}=T_{3} / T_{2}$ so the logarithmic terms cancel and an expression similar to Eq. (1) is recovered.) The effect of heat loss is to reduce $T_{2}$ and to increase $T_{4}$. Rates of heat loss will be proportional to the difference between the reservoir and environment temperatures, hence we write $\delta T_{2}=-\varepsilon\left(T_{2}-T_{0}\right)$ and $\delta T_{4}=-\varepsilon\left(T_{4}-T_{0}\right)$. The factor $\varepsilon$, which, for simplicity, is assumed the same for the HS and CS, will depend on the level of insulation and the storage duration. If, for example, the HS is designed to be at ambient temperature when discharged (as in the system proposed in ref. [4]) then $\varepsilon=0.01$ per day corresponds to a $1 \%$ loss in the stored internal energy per day. Differentiating Eq. (9) with respect to $T_{2}$ and $T_{4}$ and substituting the expressions for $\delta T_{2}$ and $\delta T_{4}$ gives:

$$
\frac{\delta B}{B}=-\varepsilon\left\{\frac{\left(T_{2}-T_{0}\right)^{2} / T_{2}+\left(T_{4}-T_{0}\right)^{2} / T_{4}}{\left(T_{2}-T_{3}\right)+\left(T_{4}-T_{1}\right)}\right\}
$$

Note that this is equivalent to the reduction in $\chi$ for an otherwise reversible PTES system.

Either $T_{1}$ or $T_{3}$ (or both) will normally be close to ambient temperature for the purpose of heat rejection. If, as is the case for both the systems proposed in refs. [4, 5], $T_{3} \approx T_{0}$, then Eq. (10) can be rearranged to give:

$$
S_{\varepsilon}=\frac{\partial \chi}{\partial \varepsilon}=-\left\{\frac{\tau-\theta}{\tau-1}+\theta \frac{\tau-1}{\tau-\theta}\right\}
$$

This equals 2 if $\theta=1$ (i.e., $T_{3}=T_{1}$, as in ref. [4]), reflecting the fact that heat losses reduce both the stored thermal energy and the efficiency with which it can be converted back into useful work.

\subsubsection{Impact of reservoir thermal irreversibility}

Krane [8] has analysed the destruction of availability within a thermal reservoir and conluded that the majority of the entering available energy would be lost. However, his analysis was for a fluid-based storage system for which much of the loss is due to mixing. Heat transfer in packed-beds (as proposed here) has received extensive attention in the literature (see, for example, ref. [9]), but little of this has focused on availability loss, which is crucial to an electricity storage scheme. Losses due to the irreversible nature of the heat transfer between the gas and storage medium are not straightforward and their magnitude depends on a variety of factors, including the mode of operation of the reservoirs. For example, if the reservoir is taken from a fully discharged to a fully charged state (the so-called single blow case), temperature gradients tend to be steep and consequently losses are high. On the other hand, periodic cycling of the reservoirs, as might be used for smoothing daily electricity demand fluctuations, yields gentler thermal gradients and lower losses, but at the expense of reduced storage capacity. Figure 4, taken from ref. [10], shows examples of these two cases. 


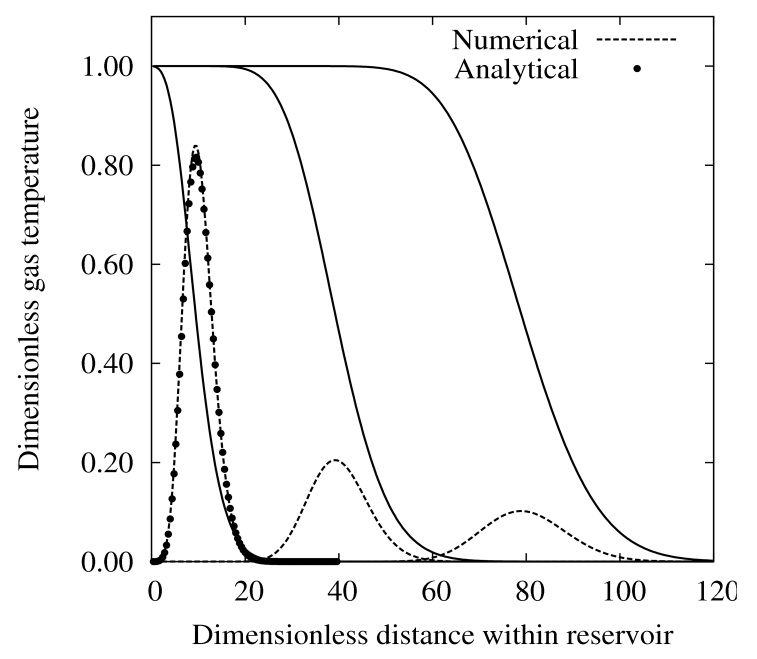

(a) single charge operation

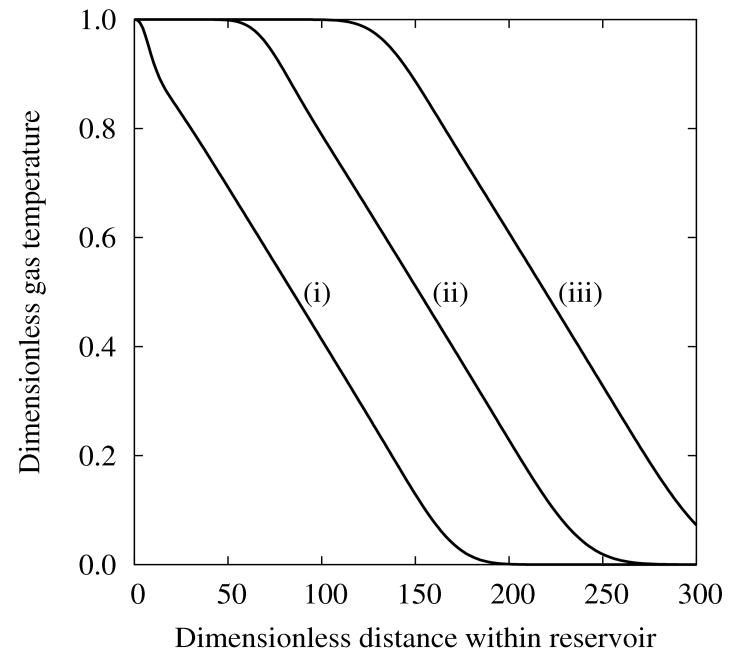

(b) cyclic operation

Figure 4: Reservoir temperature profiles for different modes of operation (taken from [10])

Single charge. The solid lines in the left-hand figure show the thermal front at three time intervals for the single charge case. The temperature profile becomes progressively less steep as it moves through the reservoir and consequently the temperature difference between gas and solid (dashed lines) gradually decreases, thereby reducing the entropy generation rate. The sloping front constitutes a loss of stored available energy and also prevents the reservoir from being fully charged without hot (or cold) gas first issuing from the exit, thereby incurring an exit loss. This means that the charged reservoir will have a non-uniform temperature distribution which will tend to equilibrate with time, thereby causing further reduction in the stored availability. Strategies to overcome the latter problem are, however, under investigation.

Cyclic operation. The shape of the temperature profiles for cyclic operation (Fig. 4b) depends on the length of the charge-discharge period relative to the time taken for an ideal (abrupt) thermal front to pass through the reservoir; in the case shown this ratio is about $50 \%$, meaning that the energy stored per cycle is only half of the maximum possible. Longer period cycles allow more energy to be stored but at the expense of steeper fronts and thus higher losses. It is worth pointing out that steady state, periodic operation necessarily incurs an exit loss, as suggested by curve (iii) in Fig. 4b which shows the situation near the end of the charge phase and indicates the temperature at the exit of the reservoir beginning to rise. The exit loss reflects the need for heat to be rejected between successive cycles in order to counter the effects of irreversible heat transfer.

Since reservoir thermal losses clearly depend on the charge-discharge history, accurate modelling can only really be undertaken by developing an overall system model that couples unsteady heat transfer calculations with thermodynamic cycle calculations, and includes the time-varying characteristics of the electrical network to which the storage system is connected. It is nonetheless possible to estimate how reservoir losses will vary with operating temperatures for a given reservoir geometry and mode of operation. The net entropy generation rate due to heat transfer between gas and solid is given by:

$$
\dot{S}=\int h \frac{\left(T_{\mathrm{g}}-T_{\mathrm{s}}\right)}{T_{\mathrm{g}} T_{\mathrm{s}}} d A
$$

where $h$ is a surface heat transfer coefficient, $T_{\mathrm{g}}$ and $T_{\mathrm{s}}$ are the local gas and solid temperatures, and the integration is carried out over the entire solid-gas interfacial area, $A$. The loss in availability is given by integrating this entropy generation rate over the charge-discharge periods and multiplying by the environment temperature, $T_{0}$. It was shown in ref. [10] that the result can be expressed in the form:

$$
\delta B=k T_{0} \times \mathrm{fn}\left(T_{C}, T_{D}\right)=-k T_{0} \Delta^{2} /\left(1+\Delta+a \Delta^{2}\right)
$$


where $T_{C}$ and $T_{D}$ are the reservoir charged and discharged temperatures, $\Delta=\left(T_{C}-T_{D}\right) / T_{D}$ and $k$ is a factor that depends on the geometry, flow conditions and mode of operation of the reservoir. The quadratic denominator of Eq. (13) stems from the inverse dependence of the entropy generation rate on $T_{\mathrm{s}} T_{\mathrm{g}}$, as shown in Eq. (12). The coefficient $a$ varies from 0 for high frequency cycles (analytical solution) to $1 / 12$ for single charge operation (numerical approximation, but with very small error). It is most likely that PTES will be used in the periodic cyclic mode and, in any case, the effect of $a$ is relatively small so it is set to zero in what follows. For simplicity, values of $k$ will be assumed equal for the hot and cold reservoirs, although in practice the higher Reynolds numbers would tend to give a larger $k$ for the CS. Substituting the cycle temperatures and normalising by the stored availability gives the following expression for the overall (fractional) availability loss:

$\xi=\frac{\delta B}{B}=-k T_{0} \frac{\left(T_{2}-T_{3}\right)^{2} / T_{2} T_{3}+\left(T_{4}-T_{1}\right)^{2} / T_{4} T_{1}}{\left(T_{2}-T_{3}\right)+\left(T_{4}-T_{1}\right)}=-2 k \frac{T_{0}}{T_{3}}\left(\frac{\tau-\theta}{\tau-1}\right)$

This expression does not include the exit loss and the loss associated with thermal equilibration during storage, but these losses are relatively small provided the reservoirs are operated sensibly [10].

\subsection{Graphical representation of sensitivity factors}

Figure 5 shows the various loss sensitivity factors (Eqs. $(6-8,11 \& 14))$ plotted as functions of the isentropic temperature ratio $\tau=T_{2} / T_{1}$. Only one line is shown for the compressor / expander heat leakage in the left hand figure since $S_{\alpha}$ is independent of $\theta$. For the reservoir losses it has been assumed that $T_{3}=T_{0}$, as discussed in section 2.3.3. Note also that the reservoir thermodynamic loss factor $\xi$ is not strictly a sensitivity factor (i.e., it is not a partial derivative of $\chi$ ), but the curves nonetheless show the relative temperature dependence of these losses; an arbitrary value of $k=1 / 2$ has been used for the results shown.

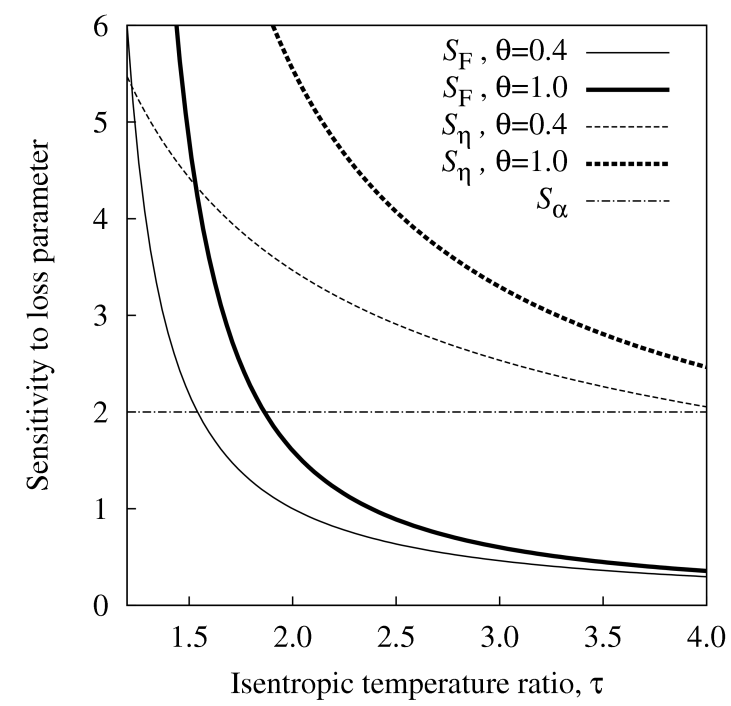

(a) cycle losses

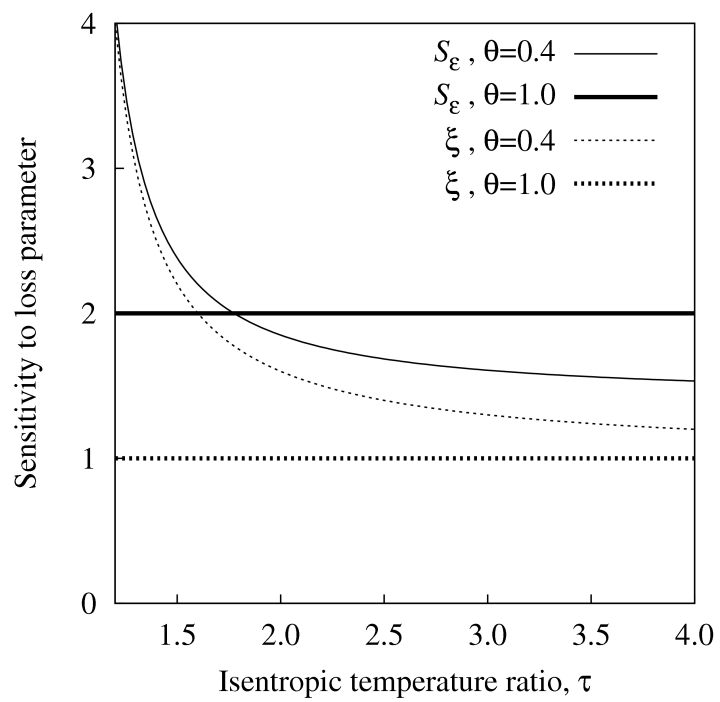

(b) reservoir losses

Figure 5: Variation of the loss sensitivity factors with isentropic temperature ratio for $\theta=1$ (bold) and $\theta=0.4$ (faint). Note that the $\xi$ curves (right-hand figure) are with $k=1 / 2$.

\section{Discussion}

The results presented in Fig. 5 demonstrate a number points which are of use in guiding research and design efforts. These may be summarised as follows:

i. The round-trip efficiency is particularly susceptible to the compression and expansion polytropic efficiency, especially at low temperature (and hence pressure) ratios. The situation is improved by reducing the ratio $\theta$, in accord with the approximate analysis based on work ratio given in section 2.2. This has been the strategy adopted for the turbomachinery-based design presented in ref. [5]. 
ii. Pressure losses seem to have a relatively small impact, provide the pressure ratio is not too low. (In addition, the values of $F$ as well as their impact will tend to be larger at lower pressure ratios, due to higher flow velocities for a given mass flow rate.)

iii. Heat leakage to and from the compressors and expanders has a very different impact to that of the polytropic efficiency. A compression heat loss during the charging phase, for example, will reduce the storage temperature (and hence reduce the stored energy), but it will also reduce the work input for the compression process. The net effect is a reduction in round-trip efficiency, but it is not as dramatic as the effect of a similar magnitude reduction in compression efficiency.

iv. The advantage of reducing $\theta$ is not so clear once reservoir losses are also taken into account. Note that both the heat loss sensitivity and thermodynamic loss factor (with $k=1$ ) take on the constant value of 2 when $\theta=1$, so reducing $\theta$ to 0.4 causes a significant increase in the thermodynamic component of loss and only marginally improves the effect of heat leakage at high pressure ratios.

\section{Conclusions}

A new method of electricity storage (PTES) has been described and aspects of its thermodynamic performance investigated, with particular focus on how various sources of loss affect the round-trip efficiency. The analysis presented has been very much simplified in order to show general trends which will help guide design. In particular, no account has been taken of how material properties (such as the thermal capacity of the storage medium) vary with temperature, and compressors and expanders have been modelled by means of simple polytropic expressions.

The round-trip efficiency and storage density both increase with the compressor temperature ratio. High temperature ratios, however, imply high pressure ratios which in turn imply high cost for the hot reservoir. This is mitigated by the use of a monatomic gas such as Argon for the working fluid. Approximate analysis also indicates that, for given compression and expansion efficiencies, it is really the ratio between the highest and lowest temperatures in each of the reservoirs $\left(R=T_{2} / T_{3}=T_{1} / T_{4}\right)$ and not the compression temperature ratio that determines the performance. Obtaining a satisfactory round-trip efficiency clearly requires highly efficient compression and expansion processes, and it is anticipated that this may be achieved by the use of reciprocating devices. For a turbomachinery-based PTES system, the effects of compression and expansion irreversibility can be mitigated by reducing the ratio between hot and cold store discharged temperatures, which also has the advantage of increasing the energy and power densities. Ultimately, however, selecting the optimal operating conditions will require reliable estimates of the various loss parameters which depend on detailed design and, in some cases, are currently subject to a degree of uncertainty. Areas in particular need of further investigation include the reservoir thermal losses and the compression and expansion efficiency for reciprocating devices.

\section{References}

[1] The UK Renewable Energy Strategy [CM 7686] DECC Publications. (2009).

[2] D.J.C. MacKay, Fluctuations and storage, in: Sustainable Energy without the Hot Air, UIT Cambridge, pp. 186-202. (2009).

[3] H. Chen, T.N. Cong, W. Yang, C. Tan, Y. Li and Y. Ding, 'Progress in electrical energy storage system: A critical review', Progress in Natural Science, 19, pp. 291-312. (2009).

[4] J. MacNaghten and J. S. Howes, 'Energy Storage', Int. Patent No. WO 2009/044139 A2. (2009).

[5] T. Desrues, J. Ruer, P. Marty and J. F. Fourmigué, 'A thermal energy storage process for large scale electric applications’, Applied Thermal Engineering. 30, pp. 425-432. (2010).

[6] A. J. White, 'Electricity storage using a thermal storage scheme', $8^{\text {th }}$ International Conference of Computational Methods in Science and Engineering (ICCMSE), Kos, Greece. (2010).

[7] A. J. White, "Thermodynamic analysis of the reverse Joule-Brayton cycle heat pump for domestic heating", Applied Energy 86 (11), pp. 2443-2450. (2009).

[8] Krane RJ. A second law analysis of the optimum design and operation of thermal energy storage systems. Int J Heat Mass Trans; 30(1) pp. 43-57. (1987).

[9] Willmott A. Dynamics of regenerative heat transfer. Taylor \& Francis; 2002.

[10] A. J. White, "Loss analysis of thermal reservoirs for electricity storage schemes", Applied Energy. 88, pp. 4150-4159. (2011). 